\title{
Entiers sans grand ni petit facteur premier I
}

\author{
par
}

\author{
ERIC SAIAS (Paris)
}

1. Introduction. Soit $\Theta(x, y, z)$ le nombre des entiers $\leq x$ et dont tous les facteurs premiers sont dans l'intervalle ]z,y]. Il apparaît doublement opportun d'étudier cette fonction. En effet, la bonne connaissance que nous avons maintenant $\mathrm{du}$ comportement asymptotique des cas particuliers $\Psi(x, y)=\Theta(x, y, 1)$ et $\Phi(x, z)=\Theta(x,+\infty, z)$ (cf. [15], III, 5 et 6 ) permet d'espérer des résultats ambitieux pour $\Theta$. D'autre part, cette fonction constitue un bon exemple de référence pour l'étude générale de la quantité $\sum_{n \leq x, p \mid n \Rightarrow p \in E} 1$, où $E$ désigne un ensemble quelconque de nombres premiers.

Tout au long de ce travail, on notera systématiquement

$$
u=\frac{\log x}{\log y} \quad \text { et } \quad v=\frac{\log x}{\log z} .
$$

On sait depuis longtemps (cf. [6], [5]) que

$$
\Psi(x, y) \sim x \varrho(u) \quad(u \ll 1 \text { et } x \rightarrow+\infty),
$$

et

$$
\Phi(x, z) \sim \frac{x \omega(v)}{\log z} \quad(v \ll 1, x \geq 2 z \text { et } x \rightarrow+\infty)
$$

où les fonctions $\varrho$ et $\omega$ sont définies par

$$
\begin{cases}\varrho(u)=1 & (0 \leq u \leq 1), \\ u \varrho^{\prime}(u)+\varrho(u-1)=0 & (u \in \mathbb{R} \backslash\{0,1\}), \\ \varrho \text { est continue en } 1 & \end{cases}
$$

et

$$
\begin{cases}v \omega(v)=1 & (1 \leq v \leq 2), \\ (v \omega(v))^{\prime}=\omega(v-1) & (v \in \mathbb{R} \backslash\{1,2\}), \\ \omega \text { est continue en } 2 . & \end{cases}
$$

En 1976, Friedlander ([8], Th. 1) a établi un résultat analogue pour 
$\Theta(x, y, z)$. Il définit $\left(^{1}\right)$ la fonction $\sigma(u, v)$ comme l'unique solution continue pour $u>1$ de l'équation

$$
u \frac{\partial \sigma}{\partial u}(u, v)+\sigma(u-1, v-v / u)=0 \quad(u>v /(v-1), v>1),
$$

avec pour conditions initiales

$$
\begin{array}{llrl}
\sigma(u, v) & =\omega(v) & & (0 \leq u<1), \\
\sigma(u, v) & =\omega(v)-1 / v & & (1 \leq u \leq v /(v-1), v>1) .
\end{array}
$$

Il démontre alors que pour

$$
1 \ll u-1 \leq v+1 \ll 1 \quad \text { et } \quad x \rightarrow+\infty,
$$

on a

$$
\Theta(x, y, z) \sim \frac{x \sigma(u, v)}{\log z} .
$$

L'un de nos objectifs est d'étendre le domaine de validité de cette dernière formule. Cela est réalisé dans [13] où nous démontrons le résultat suivant. On désigne par $\gamma$ la constante d'Euler.

ThÉORÈme A. Il existe une constante $c>0$ telle que pour tout $\varepsilon>0$, sous les conditions

$$
x \geq 3, \quad \exp \left\{(\log \log x)^{5 / 3+\varepsilon}\right\} \leq y \leq x
$$

et

on ait

$$
y \geq z^{1+c \sqrt{(\log 2 u) / u}} \geq 1
$$

$$
\Theta(x, y, z)=e^{\gamma} \prod_{p \leq z}\left(1-\frac{1}{p}\right)\left(x \sigma(u, v)+\frac{\log z}{\log y} y\right)\left(1+O_{\varepsilon}\left(\frac{\log 2 u}{\log y}\right)\right) .
$$

Comme le montre Hildebrand [9] dans le cas particulier de $\Psi(x, y)$, remplacer dans le Théorème $\mathrm{A}$ le domaine $\left(\mathrm{H}_{\varepsilon}\right)$ par une région plus grande est équivalent à améliorer le terme reste dans le Théorème des nombres premiers. Cependant on peut estimer, non plus $\Theta(x, y, z)$, mais le rapport $\Theta(x, y, z) / \Psi(x, y)$ en dehors de $\left(\mathrm{H}_{\varepsilon}\right)$. C'est l'objet du résultat suivant, démontré dans [14].

ThÉORÈme B. Sous la condition $x \geq y \geq z^{\log 2 u} \geq 1$, on a

$$
\Theta(x, y, z)=\Psi(x, y) \prod_{p \leq y}\left(1-\frac{1}{p}\right)\left(1+O\left(\frac{\log z}{\log y} \log 2 u\right)\right) .
$$

La démonstration du Théorème $\mathrm{A}$ (respectivement du Théorème $\mathrm{B}$ ) nécessite une bonne connaissance du comportement asymptotique de la

$\left({ }^{1}\right)$ En fait, la définition donnée ici pour $\sigma$ diffère de celle de Friedlander aux points $(1, v)$ avec $v \geq 1$. 
quantité $\sigma(u, v)$ (resp. $\sigma(u, v) / \varrho(u)$ ). Les résultats de Friedlander sur le sujet (cf. [8], Th. 5 et 6 ) sont ici insuffisants. L'objet de ce premier article est donc l'étude de la fonction $\sigma(u, v)$.

Je tiens ici à remercier Gérald Tenenbaum. Son apport a été très important, tant dans la conception que dans la réalisation de ce travail.

2. Nouvelle définition de $\sigma$. Avant tout, notons qu'il est naturel de prolonger $\sigma$ en une fonction définie sur $\mathbb{R}^{2}$ tout entier en posant

$$
\begin{aligned}
& \sigma(u, v)=0 \quad(u<0), \\
& \sigma(u, v)=0 \quad(u \geq 1, v \leq 1) .
\end{aligned}
$$

L'équation (3) est alors vérifiée partout, sauf aux points de discontinuité de $\sigma$ et $\partial \sigma / \partial u$.

La définition de Friedlander pour la fonction $\sigma(u, v)$ permet de montrer l'analogie qui existe entre les fonctions $\varrho, \omega$ et $\sigma$. Toutes les trois sont solutions d'équations différentielles aux différences, qui correspondent aux versions lissées des équations de Buchstab, vérifiées respectivement par $\Psi(x, y), \Phi(x, z)$ et $\Theta(x, y, z)$.

Il est important de noter que l'on peut donner une définition plus synthétique de $\sigma$, en fonction de $\varrho$ et $\omega$. De manière précise, on a

$$
\sigma(u, v)=\frac{u}{v} \varrho^{\prime}(u)+\int_{0}^{\infty} \varrho\left(u-\frac{u}{v} t\right) d \omega(t) \quad(v \geq u>0, u \neq 1) .
$$

Pour démontrer cette formule, il suffit de vérifier à l'aide des définitions de $\varrho$ et $\omega,(1)$ et $(2)$, que le membre de droite obéit aux propriétés caractérisant $\sigma$ dans la définition de Friedlander.

On sait que pour tout $u$ positif, $\sigma(u, u)=0$ (cf. [8], Th. 2(C)). En spécifiant $v=u$ dans (4), on obtient donc

$$
\varrho^{\prime}(u)+\int_{0}^{\infty} \varrho(u-t) d \omega(t)=0 \quad(u>0, u \neq 1),
$$

ce qui, en intégrant en $u$, implique

$$
\varrho(u)+\int_{0}^{\infty} \varrho(u-t) \omega(t) d t=1 \quad(u \geq 0) .
$$

On retrouve ici l'identité de convolution (cf. [15], III, 6, Exercice 1(b)) qui relie les fonctions $\varrho$ et $\omega$.

Il est intéressant de remarquer que la relation (4) est en fait la version explicitée d'une formule plus condensée qui relie de manière intime les fonctions $\varrho, \omega$ et $\sigma$. Pour décrire cette dernière formule, nous sommes amenés à introduire le langage des distributions. Nous notons dorénavant $r=u / v$. Soient $d R=\varrho d t$ et $d \Omega=\omega d t+\delta$ les solutions respectives des équations (1) 
et (2) au sens des distributions, $\delta$ désignant ici la mesure de Dirac en 0. Par le changement de variables $u=r v$, on est amené à considérer la nouvelle famille de mesures

$$
d \Omega_{r}=\omega(t / r) d t+r \delta \quad(0<r \leq 1) \quad \text { et } \quad d \Omega_{0}=e^{-\gamma} d t .
$$

On pose

$$
\sigma_{r}(u)=\sigma(u, u / r) \quad(0<r \leq 1) \quad \text { et } \quad \sigma_{0}(u)=e^{-\gamma} \varrho(u) .
$$

Les définitions de $d \Omega_{0}$ et $\sigma_{0}$ sont légitimes puisque l'on sait (cf. [3], (1.5) et [8], Th. 5) que $\lim _{v \rightarrow+\infty} \omega(v)=e^{-\gamma}$ et que pour tout $u \in \mathbb{R}, \lim _{v \rightarrow+\infty} \sigma(u, v)$ $=e^{-\gamma} \varrho(u)$. On démontre facilement (cf. [8], Th. 4) que l'on a

(5) $\quad\left(u \sigma_{r}(u)\right)^{\prime}=\sigma_{r}(u-r)-\sigma_{r}(u-1)$

$$
(0 \leq r \leq 1, u>1, u \neq 2 r, 1+r, 2) .
$$

Notons $d \Sigma_{r}=\sigma_{r} d t+r \delta$ la solution de (5) au sens des distributions. La nouvelle formule, équivalente à (4), est alors

$$
d \Sigma_{r}=(d R * d \Omega)^{\prime} .
$$

Nous achevons ce paragraphe par quelques remarques. Soit $n$ un entier positif et $r$ un réel vérifiant $0 \leq r<1$. En dérivant $n-1$ fois l'équation (5), on montre que $\sigma_{r}$ est $n$ fois continûment dérivable sur $\mathbb{R} \backslash D_{n, r}$ avec

$$
D_{n, r}=\left\{i+j r:(i, j) \in \mathbb{N}^{2}, i+j \leq n+1\right\} .
$$

De plus, $\sigma_{r}$ et toutes ses dérivées sont des fonctions réglées. Cela permet de définir chaque fonction $\sigma_{r}^{(n)}$ sur $D_{n, r}$ par continuité à droite. En dérivant $n$ fois (5), on obtient

$$
\begin{aligned}
& u \sigma_{r}^{(n+1)}(u)=\sigma_{r}^{(n)}(u-r)-\sigma_{r}^{(n)}(u-1)-(n+1) \sigma_{r}^{(n)}(u) \\
&(n \geq 0,0 \leq r \leq 1, u \in \mathbb{R}) .
\end{aligned}
$$

3. Enoncé des résultats. On utilise l'expression "sous la condition (G) ..." pour signifier : "il existe une constante $K$ telle que, sous les conditions

$$
u \geq 1 \quad \text { et } \quad v \geq u+K \sqrt{u \log 2 u},
$$

on ait ...". On note $\left(\mathrm{G}_{\eta_{1}, \eta_{2}, \ldots}\right)$ quand la constante $K$ dépend des paramètres $\eta_{1}<\eta_{2}, \ldots$

Soit $r$ un nombre réel vérifiant $0 \leq r<1$. Pour tout $u>1-r$, on désigne $\operatorname{par} \xi_{r}(u)$ l'unique solution positive $\xi$ de l'équation

$$
e^{\xi}=e^{r \xi}+u \xi .
$$

La fonction $\xi_{r}(u)$ ainsi définie est analytique pour $u>1-r$. On pose de plus $\xi_{r}(1-r)=0$. 
On sait (voir par exemple [15], III, 5.4) que la fonction implicite $\xi_{0}(u)$ permet de décrire le comportement asymptotique de $\varrho(u)=e^{\gamma} \sigma_{0}(u)$ ainsi que de toutes ses dérivées. Cela s'explique par le fait que $-\xi_{0}(u)$ est l'unique point-selle réel de la fonction de $s, \widehat{\varrho}(s) e^{u s}$. (Ici et dorénavant, on désigne par $\widehat{f}(s)$ la valeur en $s$ de la transformée de Laplace de la fonction $f$, soit

$$
\left.\widehat{f}(s)=\int_{-\infty}^{+\infty} f(t) e^{-s t} d t .\right)
$$

Cette situation se généralise à la fonction $\sigma$. Le nombre $-\xi_{r}(u)$, unique point-selle réel de la fonction $\left(\widehat{\sigma}_{r}(s)+r\right) e^{u s}$ (cf. Lemme 1(iii)), permet de décrire le comportement asymptotique de $\sigma_{r}(u)$ ainsi que de toutes ses dérivées.

ThÉORÈme 1 . Soient $n$ et $k$ des entiers $\geq 0$. Sous les conditions $u>2$ et $\left(\mathrm{G}_{n, k}\right)$, on a

$$
\text { (10) } \begin{aligned}
\sigma_{r}^{(n)}(u)= & \left(-\xi_{r}(u)\right)^{n}\left(\frac{\xi_{r}^{\prime}(u)}{2 \pi}\right)^{1 / 2} \\
& \times \exp \left\{-\int_{1-r}^{u} \xi_{r}(t) d t\right\}\left(1+f_{r, n}(u)+O_{n, k}\left(\frac{1}{u^{k}}\right)\right)
\end{aligned}
$$

où $f_{r, n}$ désigne une fonction de classe $C^{\infty}$ vérifiant

$$
f_{r, n}^{(l)}(u) \ll_{n, l} \frac{1}{u^{l+1}} \quad(l \geq 0) .
$$

L'idée d'utiliser - comme nous le faisons ici-la méthode du point-selle pour obtenir un équivalent asymptotique de $\sigma$, a été suggérée par Friedlander dans une remarque à la fin de son article [8].

En remplaçant, dans $(10), \xi_{r}(u)$ et $\xi_{r}^{\prime}(u)$ par des approximations explicites (cf. Lemme 4), on peut donner plusieurs estimations de $\sigma_{r}^{(n)}(u)$, combinant à des degrés divers simplicité et précision. On a en particulier sous la condition $(\mathrm{G})$

$$
\sigma_{r}(u)=\left(\frac{u}{1-r}\right)^{-u(1+O((\log \log 3 u) / \log 2 u))} .
$$

En utilisant (9), on montre facilement que

$$
-\int_{1-r}^{u} \xi_{r}(t) d t=-u \xi_{r}(u)+\int_{0}^{\xi_{r}(u)} \frac{e^{s}-e^{r s}}{s} d s .
$$

En substituant dans (10) et en prenant $r=n=0$ et $k=1$, on retrouve la forme usuelle de l'équivalent asymptotique de $\varrho(u)$, démontré par de Bruijn [4] et affiné par Alladi ([1], (3.9)). 
Comme nous l'avons annoncé dans l'Introduction, l'estimation asymptotique du Théorème 1 est indispensable pour établir le Théorème $\mathrm{A}$. De manière analogue, pour démontrer le Théorème $\mathrm{B}$, nous avons besoin du résultat suivant.

On désigne par $E(n, u, r)$ la quantité

$$
E(n, u, r)= \begin{cases}\frac{1}{(\log u)(u \log u)^{1-2 r}} & (u \geq n+1-r \log r, r \log u \geq 1), \\ r^{2} / u & (u \geq n+1-r \log r, r \log u<1), \\ r & (u<n+1-r \log r)\end{cases}
$$

et par $I_{r}(s)$ la fonction entière

$$
I_{r}(s)=\int_{r s}^{s} \frac{e^{w}-1}{w} d w
$$

THÉORÈme 2. Soit $n$ un entier $\geq 0$. On a pour $0 \leq r \leq 1 / 2$ et $u \geq$ $n-r \log r$

$$
\begin{aligned}
\sigma_{r}^{(n)}(u)= & \varrho^{(n)}(u) \exp \left\{-\gamma-I_{0}\left(-r \varrho^{(n+1)}(u) / \varrho^{(n)}(u)\right)\right\} \\
& \times\left(1+O_{n}(E(n, u, r))\right) .
\end{aligned}
$$

On a (cf. Lemme 8(i))

$$
-\frac{\varrho^{(n+1)}(u)}{\varrho^{(n)} u}=\xi_{0}(u)+O_{n}(1 / u)=\log u+O_{n}(\log \log 3 u) \quad(u>1) .
$$

De plus, on a $I_{0}(s) \sim s$ quand $s \rightarrow 0$ et $I_{0}(s) \sim e^{s} / s$ quand $s \rightarrow+\infty$. Cela permet de donner, suivant les cas, une estimation plus explicite de $\sigma_{r}^{(n)}(u) / \varrho^{(n)}(u)$.

On a en particulier le résultat suivant.

Corollaire. On a

$$
\sigma_{r}(u) \sim e^{-\gamma} \varrho(u) \quad(u \geq 1, v=u / r \rightarrow+\infty)
$$

si et seulement si $r \log u \rightarrow 0$.

Posons

$$
M(x, y, z)=x \prod_{z<p \leq y}\left(1-\frac{1}{p}\right)^{-1} \int_{0}^{\infty} \sigma_{r}(u-t) y^{-t} d t .
$$

La quantité $M(x, y, z)$ est une meilleure approximation de $\Theta(x, y, z)$ que $x \sigma(u, v) / \log z$ ou même que celle qui apparaît dans le Théorème $\mathrm{A}$, soit

$$
e^{\gamma} \prod_{p \leq z}\left(1-\frac{1}{p}\right)(x \sigma(u, v)+r y) \quad(\operatorname{voir}[13]) .
$$

Tant pour le Théorème $\mathrm{A}$ que pour le Théorème $\mathrm{B}$, nous avons besoin d'estimer précisément $M(x, y, z)$. C'est l'objet du résultat suivant. 
Pour $n \geq 0$ et $x \geq y>z \geq 1$, on pose

$d_{n}(x, y, z)=\max \{(i+j r) \log y-i \log \log 2 y+(1-j) \log \log 2 z:$

$$
\left.(i, j) \in \mathbb{N}^{2}, i+j \leq n+1 \text { et } i+j r<u\right\}
$$

et

$$
\Delta_{n}(x, y, z)=d_{n}(x, y, z)-\log x+(n+1) \log \log 2 y .
$$

ThÉORÈme 3 . Soit $\varepsilon$ un réel $>0$ et $n$ un entier $\geq 0$. On suppose vérifiée la condition

$$
1 \leq z<y \leq x \leq \exp \left\{y^{2 / 3-\varepsilon}\right\} .
$$

(i) Sous la condition supplémentaire $\left(\mathrm{G}_{\varepsilon}\right)$, on a

$$
M(x, y, z)=e^{\gamma} \prod_{p \leq z}\left(1-\frac{1}{p}\right)\left(x \sigma_{r}(u)+\frac{\log z}{\log y} y\right)\left(1+O_{\varepsilon}\left(\frac{\log 2 u}{\log y}\right)\right) .
$$

(ii) Sous la condition supplémentaire $\left(\mathrm{G}_{n, \varepsilon}\right)$, on a

$$
\begin{aligned}
\mid M(x, y, z)-e^{\gamma} \prod_{p \leq z}\left(1-\frac{1}{p}\right) & x \sum_{k=0}^{n} \frac{(-1)^{k} \sigma_{r}^{(k)}(u-0)}{(\log y)^{k}} \mid \\
& \ll_{n, \varepsilon}\left(1+e^{\Delta_{n}(x, y, z)}\right) \frac{x \sigma_{r}(u)(\log 2 u)^{n+1}}{(\log 2 z)(\log y)^{n+1}} .
\end{aligned}
$$

(iii) Il existe une constante $x_{0}(\varepsilon)$ telle que sous les conditions supplémentaires $\left(\mathrm{G}_{\varepsilon}\right), x \geq y^{2+\varepsilon}$ et $x \geq x_{0}(\varepsilon)$, on ait

$$
M(x, y, z)=\prod_{z<p \leq y}\left(1-\frac{1}{p}\right)^{-1} \frac{x \sigma_{r}(u)}{\log y+\sigma_{r}^{\prime}(u) / \sigma_{r}(u)}\left(1+O_{\varepsilon}\left(\frac{1}{\log x \log y}\right)\right) .
$$

Un résultat analogue au point (ii), concernant la quantité

$$
\Lambda(x, y)=\int_{0}^{\infty} \varrho(u-t) d\left(\left[y^{t}\right] / y^{t}\right)
$$

a été démontré au Lemme 4(ii) de [12].

Quand $u \geq n+1+\varepsilon$, on a $\max \left(\Delta_{n}(x, y, z), 0\right) \ll_{n, \varepsilon} 1$. Le point (ii) implique donc

$$
\begin{array}{r}
M(x, y, z) \\
=e^{\gamma} \prod_{p \leq z}\left(1-\frac{1}{p}\right) x\left[\sum_{k=0}^{n} \frac{(-1)^{k} \sigma_{r}^{(k)}(u)}{(\log y)^{k}}+O_{n, \varepsilon}\left(\frac{\sigma_{r}(u)(\log u)^{n+1}}{(\log y)^{n+1}}\right)\right], \\
\left(\left(\mathrm{L}_{\varepsilon}\right),\left(\mathrm{G}_{n, \varepsilon}\right) \text { et } u \geq n+1+\varepsilon\right) .
\end{array}
$$


4. Quelques propriétés de $\sigma_{r}$. Comme pour les transformées de fonctions, on note

$$
\widehat{\mu}(s)=\int_{-\infty}^{+\infty} e^{-t s} d \mu(t)
$$

la transformée de Laplace de la mesure de $d \mu$.

Lemme 1. Soit $r$ un réel vérifiant $0 \leq r<1$. On a

(i) $\widehat{\varrho}(s)=\exp \left\{\gamma+I_{0}(-s)\right\}(s \in \mathbb{C})$,

(ii) $\widehat{\omega}(s)=\left[s \exp \left\{\gamma+I_{0}(-s)\right\}\right]^{-1}-1(\operatorname{Re} s>0)$,

(iii) $\widehat{\sigma}_{r}(s)=\exp \left(I_{r}(-s)\right)-r(s \in \mathbb{C})$.

Dé mo n s t r a t i o n. La transformée de $\varrho$ a été calculée par Bovey (cf. [2], Lemma 1) et celle de $\omega$ par Fouvry et Tenenbaum (cf. [7], (6.14)). Montrons (iii). On obtient de manière élémentaire (cf. [15], Th. III, 5.5, (iv)) que $\varrho(t) \leq(\Gamma(t+1))^{-1}$ pour tout $t \geq 0$. Comme de plus (cf. [8], Th. 6(A)) $\sigma_{r}(t) \ll_{r} \varrho(t)$, cela montre que les transformées $\widehat{\sigma}_{r}(s)$ et $\widehat{d \Sigma_{r}}(s)$ sont bien définies pour tout $s \in \mathbb{C}$. La formule (6) permet de calculer mécaniquement ces nombres. On a en utilisant (i) et (ii)

$$
\begin{aligned}
{\widehat{d \Sigma_{r}}}_{(s)} & =\left(\left(d R * d \Omega_{r}\right)^{\prime}\right)^{\wedge}(s)=s\left(d R * d \Omega_{r}\right)^{\wedge}(s)=s \widehat{d R}(s) \widehat{d \Omega}_{r}(s) \\
& =s \exp \left\{\gamma+I_{0}(-s)\right\}\left[s \exp \left\{\gamma+I_{0}(-r s)\right\}\right]^{-1}=\exp \left(I_{r}(-s)\right) .
\end{aligned}
$$

Cela entraîne (iii).

Soient $n$ un entier $\geq 0$ et $r$ un réel vérifiant $0 \leq r<1$. Soient $a \in D_{n, r}$ et $(i, j) \in \mathbb{N}^{2}$ un couple d'entiers vérifiant

$$
a=i+j r \quad(i+j \leq n+1) .
$$

Avec ces notations, on a le

Lemme 2. $\sigma_{r}^{(n)}(a+0)-\sigma_{r}^{(n)}(a-0) \ll_{n} r^{i-n}\left(a \in D_{n, r}\right)$.

Démonstration. Posons $\Delta_{r}^{n}(a)=\sigma_{r}^{(n)}(a+0)-\sigma_{r}^{(n)}(a-0)$. On a $\Delta_{r}^{0}(r)=1$ et $\Delta_{r}^{0}(1)=-r$ pour $0 \leq r<1$. Par ailleurs, d'après $(7)$ on a

$$
a \Delta_{r}^{n+1}(a)=\Delta_{r}^{n}(a-r)-\Delta_{r}^{n}(a-1)-(n+1) \Delta_{r}^{n}(a) \quad \text { pour } n \geq 0 .
$$

En utilisant cette formule, on démontre le Lemme 2 par récurrence sur $n$. Nous omettons les détails.

Remarque. Quand $a \in D_{n, r}$, on a souvent $\Delta_{r}^{n}(a) \neq 0$. C'est par exemple le cas si $r$ est irrationnel. Ce n'est toutefois pas une règle générale, comme l'atteste l'exemple suivant : $\Delta_{1 / 3}^{7}(6)=0$.

5. Etude de $\xi_{r}$ et $I_{r}$. Pour démontrer le Théorème 1, il est agréable de disposer d'un prolongement de $\xi_{r}$ au domaine complexe. On peut en fait prolonger $\xi_{r}$ en une fonction analytique sur un ouvert simplement connexe 
de $\mathbb{C} \backslash V$ quelconque, $V$ désignant ici un voisinage de 0 suffisamment grand. Nous choisissons

$$
\mathbb{C} \backslash\left(V \cup \mathbb{R}_{-} i\right) .
$$

Ce choix permet de définir $\xi_{0}(w)$ quand $w$ est un réel négatif, ce qui est utile pour décrire le comportement asymptotique de la fonction $\omega$ (cf. [10]). Nous définissons, pour tout $w \in \mathbb{C}^{*}, \arg w$ comme l'unique réel $\left.\left.\varphi \in\right]-\pi / 2,3 \pi / 2\right]$ vérifiant $w=|w| e^{i \varphi}$. De même on définit

$$
\log w=\log |w|+i \arg w .
$$

On désignera dorénavant par $c_{0}, c_{1}, \ldots$ des constantes absolues strictement positives. On notera $c_{0}(\eta), c_{1}(\eta), \ldots$ quand ces constantes dépendront du paramètre $\eta$.

Lemme 3. Pour $0 \leq r<1, w \in \mathbb{C}$ et $|w|>c_{0}$, l'équation

$$
e^{\xi}=e^{r \xi}+w \xi
$$

admet une solution unique vérifiant

$$
\left|\xi-\xi_{r}(|w|)-i \arg w\right|<1 .
$$

On définit ainsi une fonction $\xi=\xi_{r}(w)$ qui est analytique sur

$$
\left\{w \in \mathbb{C}:|w|>c_{0}, \arg w \neq 3 \pi / 2\right\} .
$$

Démonstration. Pour $|w|>3$, on a

$$
\xi_{r}(|w|) \geq \xi_{0}(|w|)=\log |w|+\log \log |w|+O(1)
$$

d'après le Lemma 1 de [11]. De plus

$$
|w|=\frac{e^{\xi_{r}(|w|)}-e^{r \xi_{r}(|w|)}}{\xi_{r}(|w|)}=\int_{r}^{1} e^{t \xi_{r}(|w|)} d t<(1-r) e^{\xi_{r}(|w|)},
$$

d'où

$$
\xi_{r}(|w|)>\log \left(\frac{|w|}{1-r}\right)
$$

Posons

$$
\xi=\xi_{r}(|w|)+i \arg w+z .
$$

On réécrit (13) sous la forme

$$
t L(z)=z
$$

avec

$$
t=e^{(r-1) \xi_{r}(|w|)}\left(e^{i(\arg w)(r-1)}-1\right)+i|w| \arg w \cdot e^{-\xi_{r}(|w|)}
$$


et

$$
\begin{aligned}
L(z)^{-1}= & \frac{e^{z}-1}{z}-e^{(r-1)\left(\xi_{r}(|w|)+i \arg w\right)}\left(\frac{e^{r z}-1}{z}\right)-|w| e^{-\xi_{r}(|w|)} \\
= & \frac{e^{z}-1}{z}\left(1-e^{(r-1)\left(\xi_{r}(|w|)+i \arg w\right)}\right) \\
& -\left(\frac{1-e^{(1-r) z}}{z}\right) \exp \left\{r z+(r-1)\left(\xi_{r}(|w|)+i \arg w\right)\right\} \\
& -|w| e^{-\xi_{r}(|w|)} .
\end{aligned}
$$

Soit $\eta$ un paramètre assez petit. Lorsque $r \leq 1-\eta$ et $|w| \geq c_{1}(\eta)$, on a pour $|z|=1$

$$
|t| \leq 2 e^{-\eta \xi_{r}(|w|)}+\frac{3 \pi}{2}|w| e^{-\xi_{r}(|w|)} \leq \frac{1}{100},
$$

d'après (14). Par ailleurs, comme $\min _{|z|=1}\left|e^{z}-1\right| \geq 1 / 10$, on a en utilisant la première des deux formules pour $L(z)^{-1}$,

$$
\left|L(z)^{-1}\right| \geq 1 / 10-4 e^{-\eta \xi_{r}(|w|)}-|w| e^{-\xi_{r}(|w|)} \geq 1 / 50,
$$

d'après (14). D'où

$$
|t L(z)| \leq 1 / 2<|z|=1 .
$$

En appliquant le Théorème de Lagrange (cf. [16], 7.32), cela montre qu'il existe une unique solution à l'équation (16) et donc aussi à (13) sous les conditions $|w| \geq c_{1}(\eta)$ et $w \notin \mathbb{R}_{-} i$. De plus, cette solution $\xi=\xi_{r}(w)$ est analytique en $w$.

Nous pouvons donc supposer maintenant $1-\eta<r<1$ avec $\eta$ choisi arbitrairement petit. On a d'après (15)

Cela implique

$$
1-e^{-(1-r) \xi_{r}(|w|)} \gg(1-r) \log \frac{1}{1-r} .
$$

$$
\begin{aligned}
\left|1-e^{(r-1)\left(\xi_{r}(|w|)+i \arg w\right)}\right| & =\left|1-e^{-(1-r) \xi_{r}(|w|)}(1+O(1-r))\right| \\
& \geq c_{2}(1-r) \log \left(\frac{1}{1-r}\right)-c_{3}(1-r) \\
& \geq c_{4}(1-r) \log \left(\frac{1}{1-r}\right)
\end{aligned}
$$

pour $\eta$ convenablement choisi. En reportant dans la seconde expression de $L(z)^{-1}$, on obtient lorsque $|z|=1$,

$$
\left|L(z)^{-1}\right| \geq \frac{c_{5}}{10}(1-r) \log \left(\frac{1}{1-r}\right)-c_{6}(1-r) \geq c_{7}(1-r) \log \left(\frac{1}{1-r}\right)
$$

pourvu que $\eta$ soit suffisamment petit. Par ailleurs, on a clairement

$$
|t| \leq c_{8}(1-r) .
$$


Donc pour $\eta$ convenablement choisi, on a

$$
|t L(z)| \leq \frac{c_{8}}{c_{7} \log (1 /(1-r))} \leq \frac{1}{2}<|z|=1,
$$

ce qui permet de conclure comme précédemment avec le Théorème de Lagrange.

Lemme 4. Pour $0 \leq r<1,|w|>c_{9}$ et $n \geq 1$, on a

$$
\begin{gathered}
\operatorname{Re} \xi_{r}(w)>\log \left(\frac{|w|}{1-r}\right), \\
-\pi / 2-1<\operatorname{Im} \xi_{r}(w)<3 \pi / 2+1,
\end{gathered}
$$

(v) $\quad \xi_{r}(w)=\xi_{0}(w)+O\left(\frac{\min (1, r \log |w|)}{(w \log w)^{1-r}}\right) \quad((1-r) \log |w| \geq 1)$,

(vi) $\quad \xi_{r}(w)=(1+O(1-r)) \log \left(\frac{w}{1-r}\right) \quad((1-r) \log |w|<1)$,

(vii) $\quad \xi_{r}^{(n)}(w)=\frac{(-1)^{n+1}(n-1) !}{w^{n}}\left(1+O_{n}\left(\frac{1}{\log (w /(1-r))}\right)\right)$.

Démonstration. Pour alléger l'écriture, on notera parfois $\xi$ pour $\xi_{r}(w)$. On a

$$
|w|=\left|\frac{e^{\xi}-e^{r \xi}}{\xi}\right|=\left|\int_{r}^{1} e^{\xi} d \xi\right|<(1-r) e^{\operatorname{Re} \xi}
$$

et

$$
\begin{aligned}
|w| & =\left|\frac{e^{\xi}-e^{r \xi}}{\xi}\right| \geq \frac{e^{\operatorname{Re} \xi}-e^{r \operatorname{Re} \xi}}{|\xi|} \\
& =\frac{\operatorname{Re} \xi}{|\xi|} \int_{r}^{1} e^{t \operatorname{Re} \xi} d t>\frac{\operatorname{Re} \xi}{|\xi|}(1-r) e^{r \operatorname{Re} \xi}
\end{aligned}
$$

Cela montre les points (i) et (iii). Le point (ii) découle directement du Lemme 3. Le point (iv) se démontre comme la formule (3.6) de [10]. Il faut toutefois être conscient du fait que nous n'utilisons pas ici la même détermination du logarithme que dans [10]. Montrons (vi). L'équation (13) peut encore s'écrire

$$
e^{\xi}\left(1-e^{-(1-r) \xi}\right)=w \xi
$$


Cela entraîne que si $(1-r) \operatorname{Re} \xi>1$, on a $e^{\xi} \asymp w \xi$ et $\xi \ll \log w$. Donc sous l'hypothèse $(1-r) \log |w|<1$, on a toujours $(1-r) \xi \ll 1$ et d'après (ii), $|(1-r) \operatorname{Im} \xi|<3 \pi / 2+1<2 \pi$. D'où $\xi=\log (w /(1-r))+O(\min (1,(1-r) \xi))$, ce qui nous permet de conclure la démonstration de (vi). En dérivant (13), on obtient

$$
\left((1-r) e^{r \xi}+(\xi-1) w\right) \xi^{\prime}=\xi .
$$

Les points (i) et (iii) montrent que le facteur de $\xi^{\prime}$ dans cette dernière formule est égal à $\xi\left(1+O\left(\log ^{-1}(w /(1-r))\right)\right)$. Cela permet de démontrer le point (vii) pour $n=1$. L'utilisation de la formule de Cauchy

$$
\xi_{r}^{(n)}(w)=(n-1) ! \int_{|s-w|=|w| / 2} \frac{\xi_{r}^{\prime}(s)}{(s-w)^{n}} d s
$$

permet alors de montrer (vii) pour tout $n \geq 1$.

Il reste à montrer le point $(\mathrm{v})$. Pour cela on suppose donc

$$
(1-r) \log |w| \geq 1 \text {. }
$$

Si $r \gg 1$ et $w \ll 1$, il suffit de montrer que $\xi \ll 1$, ce qui se fait en utilisant la continuité sur un compact de la fonction des deux variables $w$ et $r, \xi_{r}(w)$. On pose

$$
h=\frac{e^{r \xi_{r}(w)}-1}{\xi_{r}(w)} .
$$

Avec $c_{10}$ et $c_{11}$ convenablement choisis, pour $r<c_{10}$ ou $|w|>c_{11}$, on a

$$
|h|<|w|-1 \text {. }
$$

En effet, si $r<c_{10}$, cela découle de (17), et si $|w|>c_{11}$, en appliquant successivement les points (iii), (i) et l'inégalité (18), on a

$$
\begin{aligned}
\left|\frac{e^{r \xi_{r}(w)}}{\xi_{r}(w)}\right| & <\frac{|w|}{(1-r) \operatorname{Re} \xi_{r}(w)}<\frac{|w|}{(1-r) \log (|w| /(1-r))} \\
& \leq \frac{|w|}{1+(\log 2) / \log |w|} .
\end{aligned}
$$

On a $e^{\xi_{r}(w)}=1+(w+h) \xi_{r}(w)$. En utilisant le Lemme 3 avec $r=0$ et $(20)$, on en déduit que pour $w$ réel $>c_{11}$, on a

$$
\xi_{r}(w)=\xi_{0}(w+h) .
$$

Par prolongement analytique, cela reste vrai pour tout $w \in \mathbb{C}$ tel que $|w|>$ $c_{11}$. En appliquant (vii) avec $n=2$, on en déduit que

$$
\xi_{r}(w)=\xi_{0}(w)+h \xi_{0}^{\prime}(w)+O\left(h^{2} / w^{2}\right),
$$


soit en particulier $\xi_{r}(w)=\xi_{0}(w)+O(1)$. En reportant cette dernière estimation de $\xi_{r}(w)$ dans (19) et en utilisant (iv) on obtient

$$
h \ll \frac{w^{r} \min (1, r \log |w|)}{(\log w)^{1-r}} .
$$

Pour conclure, il suffit de reporter cette dernière majoration dans (21).

Lemme 5. Soient $r$ et $u$ des réels vérifiant $0 \leq r<1$ et $u \geq 1$. Soient $s$ et $\tau$ des complexes vérifiant $s=\xi_{r}(u)+i \tau$. Soit $n$ un entier $\geq 0$. On a

$$
\begin{gathered}
\text { (i) } \quad I_{r}^{(n)}(s)=u\left(1+O_{n}\left(\frac{1}{\log (2 u /(1-r))}+|\tau|\right)\right) \quad(\tau \ll 1), \\
\text { (ii) } \quad\left|e^{I_{r}(s)}\right| \leq \exp \left\{I_{r}\left(\xi_{r}(u)\right)-c_{12} \tau^{2} u\right\} \quad(\tau \in \mathbb{R},|\tau| \leq \pi), \\
\text { (iii) }\left|e^{I_{r}(s)}\right| \leq \exp \left\{I_{r}\left(\xi_{r}(u)\right)-c_{13} u \min ^{2}\left(1-r,(\log u)^{-1}\right)\right\} \\
\end{gathered}
$$

$e^{I_{r}(s)}=-\frac{e^{-\gamma}}{s}\left(1+O\left(\frac{e^{\xi}}{\tau}+r \tau\right)\right) \quad\left(e^{\xi} \leq|\tau| \ll r^{-1}\right)$

$e^{I_{r}(s)}=r\left(1+O\left(\frac{e^{\xi}}{\tau}+\frac{e^{r \xi}}{r \tau}\right)\right) \quad\left(|\tau| \geq e^{\xi}, r \tau \gg 1\right)$.

Démonstration. Si $r<1 / 2$, on a

$$
I_{r}(\xi)=\int_{r \xi}^{\xi} \frac{e^{t}-1}{t} d t=\frac{e^{\xi}}{\xi}\left(1+O\left(\frac{1}{\xi}\right)\right)=u\left(1+O\left(\frac{1}{\log 2 u}\right)\right)
$$

d'après les points (v) et (vi) du Lemme 4. Si $r \geq 1 / 2$, on a $I_{r}(\xi)=u+A$ avec

$$
A=\int_{r}^{1}\left(\frac{e^{t \xi}-1}{t}-e^{t \xi}\right) d t \ll 1-r+\int_{r}^{1}(1-t) e^{t \xi} d t \ll \frac{u}{\log (2 u /(1-r))} .
$$

Donc

$$
I_{r}(\xi)=u\left(1+O\left(\frac{1}{\log (2 u /(1-r))}\right)\right) .
$$

Par définition de $\xi$, on a

$$
I_{r}^{\prime}\left(\xi_{r}(u)\right)=u
$$

et donc

$$
I_{r}^{\prime \prime}\left(\xi_{r}(u)\right)=1 / \xi_{r}^{\prime}(u)
$$

En dérivant cette dernière identité, on obtient une expression de $I_{r}^{(n)}(\xi)$ en fonction des dérivées de $\xi_{r}(u)$ qui permet par récurrence, et à l'aide du 
Lemme 4(vii), de montrer que

Pour $\tau \ll 1$, on a donc

$$
I_{r}^{(n)}(\xi)=u\left(1+O_{n}\left(\frac{1}{\log (2 u /(1-r))}\right)\right) \quad \text { pour } n \geq 1 .
$$

$$
I_{r}^{(n)}(s)=\int_{r}^{1} e^{t s} t^{n-1} d t \ll \int_{r}^{1} e^{t \xi} t^{n-1} d t=I_{r}^{(n)}(\xi) \ll_{n} u
$$

et par la formule des accroissements finis

$$
I_{r}^{(n)}(s)=I_{r}^{(n)}(\xi)+O_{n}(u \tau)=u\left(1+O_{n}\left(\frac{1}{\log (2 u /(1-r))}+|\tau|\right)\right) .
$$

Cela démontre (i).

On suppose dorénavant $\tau \in \mathbb{R}$. On a

$$
\left|e^{I_{r}(s)}\right| \leq \exp \left\{I_{r}(\xi)-H(\tau)\right\}
$$

avec

$$
H(\tau)=H_{r, u}(\tau)=\int_{r}^{1} \frac{e^{t \xi}-1}{t}(1-\cos t \tau) d t .
$$

On va obtenir (ii) et (iii) en minorant convenablement $H(\tau)$. Pour $0 \leq t \leq 1$ et $|\tau| \leq \pi$, on a $1-\cos t \tau \geq 2 t^{2} \tau^{2} / \pi^{2}$, d'où

$$
H(\tau) \geq \frac{2 \tau^{2}}{\pi} \int_{r}^{1}\left(e^{t \xi}-1\right) t d t \gg \tau^{2} I_{r}^{\prime \prime}(\xi) \gg \tau^{2} u .
$$

Cela démontre (ii). Par la seconde formule de la moyenne on a

$$
\int_{r}^{1} \frac{e^{t \xi}-1}{t} \cos t \tau d t \leq \frac{2\left(e^{\xi}-1\right)}{|\tau|},
$$

d'où, pour $|\tau| \geq \pi$ et $k$ entier positif,

$$
\begin{aligned}
H(k \tau) & =\int_{r}^{1}\left(\frac{e^{t \xi}-1}{t}\right)(1-\cos k \tau t) d t \\
& \geq \int_{r}^{1} \frac{e^{t \xi}-1}{t} d t-\frac{2\left(e^{\xi}-1\right)}{k|\tau|} \gg u\left(1-\frac{c_{14} e^{\xi}}{k u}\right) .
\end{aligned}
$$

Par ailleurs, on remarque que l'inégalité $\left|1-e^{i k \tau t}\right|^{2} \leq k^{2}\left|1-e^{i \tau t}\right|$ entraîne $H(k \tau) \leq k^{2} H(\tau)$. En choisissant $k=1+\left[2 c_{14} e^{\xi} / u\right]$, on obtient donc

$$
H(\tau) \geq \frac{H(k \tau)}{k^{2}} \gg \frac{u}{k^{2}} \asymp \frac{u^{3}}{e^{2 \xi}} .
$$

Les formules (v) et (vi) du Lemme 4 permettent maintenant de conclure la démonstration du point (iii) du Lemme 5 . 
Les estimations de $e^{I_{r}(s)}$ des points (iv) et (v) se démontrent à partir de l'expression $e^{I_{r}(s)}=e^{I_{0}(s)-I_{0}(r s)}$ avec (cf. Lemme 7 de [12])

$$
I_{0}(s)=-\log (-s)-\gamma+O\left(e^{s} / s\right) \quad\left(s \in \mathbb{C} \backslash \mathbb{R}_{+}\right) .
$$

Dans cette formule, le symbole Log désigne la branche principale du logarithme.

On estime $e^{-I_{0}(r s)}$ par l'intermédiaire de la formule élémentaire

$$
I_{0}(r s) \ll r s \quad(r s \ll 1)
$$

pour (iv) et de la formule (24) pour (v).

Cela achève la démonstration du Lemme 5.

Nous désignons dorénavant $\operatorname{par} \varphi_{k, l}(u)(l=0,1,2, \ldots)$ une fonction $C^{\infty}$ de $u$ paramétrée par le réel $r$, qui vérifie

$$
\frac{d^{j} \varphi_{k, l}(u)}{d u^{j}} \ll_{j, n} \frac{1}{u^{j+k}} \quad(j \geq 0)
$$

uniformément en $u$ et $r$. On désigne par $\mathcal{F}_{k}$ l'ensemble de ces fonctions. On note $\varphi_{k, l}(u, \alpha)$ pour désigner une éventuelle dépendance en le paramètre supplémentaire $\alpha$.

Lemme 6. Sous les conditions $0 \leq r<1, w>0$ et $u>w+c_{9}$, on a

$$
\begin{aligned}
\exp \left\{\int_{u-w}^{u} \xi_{r}(t) d t\right\} & =e^{w \xi_{r}(u)}\left(1-\frac{w^{2}}{2} \xi_{r}^{\prime}(u)+w^{3} \varphi_{2,0}(u, w)\right) \\
& =e^{w \xi_{r}(u)} \varphi_{0,1}(u, w) .
\end{aligned}
$$

Démonstration. Par la formule de Taylor avec reste intégral, on a

$$
\begin{aligned}
\int_{u-w}^{u} \xi_{r}(t) d t & =w \xi_{r}(u)-\frac{w^{2}}{2} \xi_{r}^{\prime}(u)-\frac{1}{2} \int_{0}^{w} t^{2} \xi_{r}^{\prime \prime}(u-w+t) d t \\
& =w \xi_{r}(u)-\frac{w^{2}}{2} \xi_{r}^{\prime}(u)+w^{3} \varphi_{2,2}(u, w)
\end{aligned}
$$

d'après le Lemme 4(vii). En prenant l'exponentielle de cette formule, on en déduit la relation voulue.

6. Démonstration du Théorème 1. Nous procédons par récurrence sur l'entier $n \geq 0$. Nous examinons donc en premier lieu le cas $n=0$. Supposons dans un premier temps que $2<u \leq c_{9}$. D'après le Lemme 4 , on a alors sous la condition supplémentaire $(\mathrm{G}), \xi_{r}(u) \asymp 1$. D'après $(23)$, on a aussi $\xi_{r}^{\prime}(u) \asymp 1$. L'énoncé du Théorème 1 est donc ici équivalent à l'assertion $\sigma(u, v) \ll 1$. Or la fonction $\omega$ est bornée (voir par exemple [15], III, $6,(22))$. Donc $\sigma(u, v)$ est également bornée pour $u \leq v /(v-1)$. Comme $\sigma \geq 0$ (cf. [8], Th. 2), d'après (3) $\sigma(u, v)$ est décroissante en $u$, à $v$ fixé. On 
en déduit que $\sigma(u, v)$ est bornée sur tout $\mathbb{R}^{2}$. On suppose donc maintenant $u>c_{9}$.

En appliquant la formule d'inversion de Laplace sur la droite d'abscisse $-\xi=-\xi_{r}(u)$, on obtient

$$
2 i \pi \sigma_{r}(u)=\int_{-\xi-i \infty}^{-\xi+i \infty} \widehat{\sigma}_{r}(s) e^{u s} d s=\int_{\xi-i \infty}^{\xi+i \infty}\left(e^{I_{r}(s)}-r\right) e^{-u s} d s
$$

d'après le Lemme 1(iii).

Examinons cette dernière expression de $\sigma_{r}(u)$. La lettre $s$ désignant un nombre complexe, les réels $\kappa$ et $\tau$ seront dorénavant implicitement définis par la relation $s=\kappa+i \tau$. On a

$$
-r \int_{\kappa=\xi,|\tau| \leq e^{\xi}} e^{-u s} d s \ll e^{-u \xi} .
$$

D'autre part, en choisissant convenablement la constante $K(k)$ qui intervient dans la définition (8) de $\left(\mathrm{G}_{k}\right)$, on a par le Lemme 5(ii) et (iii)

$$
\int_{\kappa=\xi, u^{-1 / 3} \leq|\tau| \leq e^{\xi}} e^{I_{r}(s)-u s} d s \ll \frac{\exp \left\{I_{r}(\xi)-u \xi\right\}}{u^{1+k}} \quad\left(\left(\mathrm{G}_{k}\right)\right) .
$$

Enfin, par une intégration par parties, on a

$$
\begin{aligned}
J & =\int_{\kappa=\xi,|\tau| \geq e^{\xi}}\left(e^{I_{r}(s)}-r\right) e^{-u s} d s \\
& =\frac{i}{u}\left[\left(e^{I_{r}(\xi-i \tau)}-r\right) e^{-u(\xi-i \tau)}\right]_{\tau=-e^{\xi}}^{\tau=e^{\xi}}+\frac{i}{u} \int_{\kappa=\xi,|\tau| \geq e^{\xi}} \frac{e^{r s}-e^{s}}{s} e^{I_{r}(s)-u s} d s .
\end{aligned}
$$

En utilisant le Lemme 5(iv) et (v), il suit

$$
J \ll e^{-u \xi} .
$$

En réunissant les majorations (25), (26) et (27), on obtient

$$
\sigma_{r}(u)=\frac{1}{2 i \pi} \int_{\xi-i u^{-1 / 3}}^{\xi+i u^{-1 / 3}} e^{I_{r}(s)-u s} d s+O\left(\frac{e^{I_{r}(\xi)-u \xi}}{u^{1+k}}\right) \quad\left(\left(\mathrm{G}_{k}\right)\right) .
$$

Par (11) et le Lemme 4, on voit que le terme d'erreur ci-dessus est convenable. Il reste à examiner le terme principal.

Par la formule de Taylor avec reste intégral, on a

$$
I_{r}(s)=I_{r}(\xi)+i \tau I_{r}^{\prime}(\xi)-\frac{\tau^{2}}{2} I_{r}^{\prime \prime}(\xi)+K_{r}(u, \tau)
$$


avec

$$
K_{r}(u, \tau)=-\frac{i}{2} \int_{0}^{\tau}(\tau-t)^{2} I_{r}^{\prime \prime \prime}(\xi+i t) d t .
$$

En utilisant (22), (23) et le Lemme 5(i) avec $n=2$, on obtient donc

$$
\begin{aligned}
\frac{1}{2 i \pi} \int_{\xi-i u^{-1 / 3}}^{\xi+i u^{-1 / 3}} e^{I_{r}(s)-u s} d s & \\
& =\frac{e^{-u \xi+I_{r}(\xi)}}{2 \pi} \int_{-u^{-1 / 3}}^{u^{-1 / 3}} \exp \left\{-\frac{\tau^{2}}{2} I_{r}^{\prime \prime}(\xi)+K_{r}(u, \tau)\right\} d \tau \\
& =\left(\frac{\xi_{r}^{\prime}(u)}{2 \pi}\right)^{1 / 2} e^{-u \xi+I_{r}(\xi)}\left(1+H_{r}(u)+O\left(e^{-u^{1 / 4}}\right)\right)
\end{aligned}
$$

avec

$$
H_{r}(u)=\left(\frac{\xi_{r}^{\prime}(u)}{2 \pi}\right)^{1 / 2} \int_{-u^{-1 / 3}}^{u^{-1 / 3}} \exp \left\{-\frac{\tau^{2}}{2} I_{r}^{\prime \prime}(\xi)\right\}\left(e^{K_{r}(u, \tau)}-1\right) d \tau .
$$

Pour conclure la démonstration du cas $n=0$ du Théorème 1 , il reste à montrer, au regard de $(28)$, que $H_{r}$ est élément de $\mathcal{F}_{1}$. Pour cela, nous allons utiliser le résultat suivant.

LEMme 7. Soit $T(w)$ une fonction analytique pour $\operatorname{Re} w>c_{15}$. On suppose que

$$
T(w) \ll 1 / u \quad\left(u>2 c_{15},|u-w| \leq u / 2\right) .
$$

On a alors

$$
T^{(k)}(u) \ll_{k} 1 / u^{1+k} \quad\left(k \geq 0, u>2 c_{15}\right) .
$$

Dém on stration. La démonstration est immédiate en utilisant la formule de Cauchy. On a

$$
T^{(k)}(u)=k ! \int_{|w-u|=u / 2} \frac{T(w)}{(w-u)^{k+1}} d w \ll_{k} \frac{1}{u^{k+1}} .
$$

Revenons au cas spécifique de $H_{r}$. Par le Lemme 3 , la fonction $\xi_{r}(w)$ est analytique sur $\operatorname{Re} w>c_{0}$. De plus la fonction $I_{r}$ est entière. Donc en considérant la détermination continue de $w^{-1 / 3}$ qui est réelle quand $w$ est réel, on voit que $H_{r}(w)$ est analytique sur $\operatorname{Re} w>c_{0}$. Par le Lemme 4 et le Lemme 5(i), on a pour $\operatorname{Re} w>c_{9}$ et $|\tau| \leq 1$

$$
K_{r}(w, \tau)=-i I_{r}^{\prime \prime \prime}\left(\xi_{r}(w)\right) \frac{\tau^{3}}{6}+O\left(u \tau^{4}\right) .
$$


On en déduit que $H_{r}(w) \ll 1 / u$ pour Re $w>c_{9}$. Le Lemme 7 permet alors de conclure que $H_{r}$ est bien élément de $\mathcal{F}_{1}$.

Nous passons maintenant à la partie inductive de la démonstration par récurrence. Nous supposons donc le Théorème 1 démontré pour l'entier $n \geq 0$ et nous proposons de le montrer pour $n+1$. Le point essentiel de la démonstration est maintenant l'utilisation de la formule de récurrence (7) qui exprime $\sigma_{r}^{(n+1)}(u)$ en fonction de $\sigma_{r}^{(n)}(u)$. Voyons cela.

Posons

$$
F(u)=F_{r}(u)=\exp \left\{-\int_{1-r}^{u} \xi_{r}(t) d t\right\}
$$

et

$$
G_{n}(u)=G_{r, n}(u)=\left(\frac{\xi_{r}^{\prime}(u)}{2 \pi}\right)^{1 / 2}\left(-\xi_{r}(u)\right)^{n} .
$$

Conformément aux notations du Théorème 1, notre objectif est de montrer que

$$
\begin{aligned}
\sigma_{r}^{(n+1)}(u)=F(u) G_{n+1}(u)\left(1+f_{r, n+1}(u)\right. & \left.+O_{n, k}\left(1 / u^{k}\right)\right) \\
& \left(k \geq 0, u>2 \text { et }\left(\mathrm{G}_{n, k}\right)\right)
\end{aligned}
$$

avec de plus

$$
f_{r, n+1}(u)=f_{r, n}(u)-\frac{\xi_{r}^{\prime \prime}(u)}{2 \xi_{r}^{\prime}(u) \xi_{r}(u)}-n \frac{\xi_{r}^{\prime}(u)}{\left(\xi_{r}(u)\right)^{2}}+\frac{\varphi_{1,3}(u, n)}{\sqrt{u}} .
$$

Pour simplifier l'écriture, on notera souvent $f_{n}(u)$ à la place de $f_{r, n}(u)$. D'après (7) et l'hypothèse de récurrence, on a

avec

$$
u \sigma_{r}^{(n+1)}(u)=\sum_{i=0}^{4} A_{i}
$$

$$
\begin{aligned}
& A_{0}=\left(1+f_{n}(u-r)\right) G_{n}(u-1)(F(u-r)-F(u-1)), \\
& A_{1}=\left(1+f_{n}(u-r)\right) F(u-r)\left(G_{n}(u-r)-G_{n}(u-1)\right), \\
& A_{2}=-(n+1)\left(1+f_{n}(u)\right) F(u) G_{n}(u), \\
& A_{3}=\left(f_{n}(u-r)-f_{n}(u-1)\right) F(u-1) G_{n}(u-1), \\
& A_{4}=O_{n, k}\left(\frac{F(u-1) G_{n}(u)}{u^{k}}\right) .
\end{aligned}
$$

Par le Lemme 4, on a

$$
\xi_{r}(u) \leq \frac{3}{2} \log u+O(1) \quad(u>1-r,(\mathrm{G}))
$$

Avec le Lemme 6 , cela entraîne que $A_{4} \ll_{n, k} F(u) G_{n}(u) u^{2-k}$, ce qui est un terme reste convenable. En utilisant de plus que $f_{n} \in \mathcal{F}_{1}$, on a par le Lemme $7, A_{3}=F(u) G_{n}(u) \sqrt{u} \varphi_{1,4}(u, n)$, ce qui est également convenable. 
Le Lemme 7 sera dorénavant implicitement utilisé à chaque fois que l'on affirmera qu'une fonction appartient à l'un des $\mathcal{F}_{k}$. On a

$$
A_{2}=-F(u) G_{n}(u)\left(n+1+\varphi_{1,5}(u, n)\right) .
$$

Par ailleurs, on a

$$
\frac{G_{n}(u-r)-G_{n}(u-1)}{G_{n}(u)}=(1-r)\left(\frac{\xi_{r}^{\prime \prime}(u)}{2 \xi_{r}^{\prime}(u)}+n \frac{\xi_{r}^{\prime}(u)}{\xi_{r}(u)}\right)+\varphi_{2,6}(u, n) .
$$

Par le Lemme 6 et la formule (31), on obtient donc

(33) $A_{1}=F(u) G_{n}(u)\left[e^{r \xi_{r}(u)}(1-r)\left(\frac{\xi_{r}^{\prime \prime}(u)}{2 \xi_{r}^{\prime}(u)}+n \frac{\xi_{r}^{\prime}(u)}{\xi_{r}(u)}\right)+\frac{\varphi_{0,7}(u, n)}{\sqrt{u}}\right]$.

Enfin on a

$$
G_{n}(u-1)=G_{n}(u)\left(1-\frac{\xi_{r}^{\prime \prime}(u)}{2 \xi_{r}^{\prime}(u)}+n \frac{\xi_{r}^{\prime}(u)}{\xi_{r}(u)}+\varphi_{2,8}(u, n)\right)
$$

et par le Lemme 6 et l'inégalité (31),

$$
\begin{aligned}
& \frac{F(u-r)-F(u-1)}{F(u)} \\
& \quad=e^{r \xi_{r}(u)}\left(1-\frac{r^{2}}{2} \xi_{r}^{\prime}(u)\right)-e^{\xi_{r}(u)}\left(1-\frac{\xi_{r}^{\prime}(u)}{2}\right)+\sqrt{u} \varphi_{1,9}(u) \\
& \quad=-u \xi_{r}(u)\left(1-\frac{\xi_{r}^{\prime}(u)}{2}\right)+e^{r \xi_{r}(u)} \frac{1-r^{2}}{2} \xi_{r}^{\prime}(u)+\sqrt{u} \varphi_{1,9}(u)
\end{aligned}
$$

d'après (9). On a

$$
\begin{aligned}
\frac{A_{0}}{u}=F(u) G_{n+1}(u)\left[1+f_{n}(u)-\frac{1}{2}\left(\xi_{r}^{\prime}(u)+\frac{\xi_{r}^{\prime \prime}(u)}{\xi_{r}^{\prime}(u)}+\frac{\left(1-r^{2}\right) e^{r \xi_{r}(u)} \xi_{r}^{\prime}(u)}{u \xi_{r}(u)}\right)\right. \\
\left.-n \frac{\xi_{r}^{\prime}(u)}{\xi_{r}(u)}+\frac{\varphi_{1,10}(u, n)}{\sqrt{u}}\right] .
\end{aligned}
$$

D'où avec (32) et (33)

$$
\begin{aligned}
\sigma_{r}^{(n+1)}(u)= & F(u) G_{n+1}(u)\left[1+f_{n}(u)\right. \\
& -\frac{1}{2 u \xi_{r}(u)}\left(-2+(1-r) e^{r \xi_{r}(u)} \frac{\xi_{r}^{\prime \prime}(u)}{\xi_{r}(u)}+u \xi_{r}(u) \xi_{r}^{\prime}(u)\right. \\
& \left.+\frac{u \xi_{r}(u) \xi_{r}^{\prime \prime}(u)}{\xi_{r}^{\prime}(u)}+\left(1-r^{2}\right) e^{r \xi_{r}(u)} \xi_{r}^{\prime}(u)\right) \\
& +\frac{n}{u \xi_{r}(u)}\left(1-\frac{(1-r) e^{r \xi_{r}(u)} \xi_{r}^{\prime}(u)}{\xi_{r}(u)}-u \xi_{r}^{\prime}(u)\right) \\
& \left.+\frac{\varphi_{1,11}(u, n)}{\sqrt{u}}+O_{n, k}\left(\frac{1}{u^{k}}\right)\right] .
\end{aligned}
$$


En dérivant (13), on obtient

$$
\xi_{r}^{\prime}(u)\left((1-r) e^{r \xi_{r}(u)}+u\left(\xi_{r}(u)-1\right)\right)=\xi_{r}(u) .
$$

Donc la deuxième expression entre parenthèses dans le membre de droite de (34) est $=-u \xi_{r}^{\prime}(u) / \xi_{r}(u)$ et la première expression entre parenthèses est

$$
=(1-r) e^{r \xi_{r}(u)}\left(\frac{\xi_{r}^{\prime \prime}(u)}{\xi_{r}^{\prime}(u)}+r \xi_{r}^{\prime}(u)\right)-2+\frac{u \xi_{r}(u) \xi_{r}^{\prime \prime}(u)}{\xi_{r}^{\prime}(u)}+\xi_{r}(u)+u \xi_{r}^{\prime}(u) .
$$

En dérivant deux fois (13), on voit que cette dernière expression est $=$ $u \xi_{r}^{\prime \prime}(u) / \xi_{r}^{\prime}(u)$

Cela conclut la démonstration de (29) et (30), et partant celle du Théorème 1 .

Le résultat suivant découle facilement des formules (29) et (30).

Lemme 8. Soit $n$ un entier $\geq 0$. Sous les conditions $\left(\mathrm{G}_{n}\right)$ et $u>c_{16}(n)$, on $a$

$$
\begin{gathered}
\frac{\sigma_{r}^{(n+1)}(u)}{\sigma_{r}^{(n)}(u)}=-\xi_{r}(u)+O_{n}\left(\frac{1}{u}\right), \\
\frac{d}{d u} \frac{\sigma_{r}^{(n+1)}(u)}{\sigma_{r}^{(n)}(u)}=-\xi_{r}^{\prime}(u)+O_{n}\left(\frac{(\log u)^{2}}{u^{3 / 2}}\right) .
\end{gathered}
$$

Démonstration. Supposons $n \geq 0$ fixé. D'après le Théorème 1 , on a pour $u$ suffisamment grand

$$
\frac{\sigma_{r}^{(n+1)}(u)}{\sigma_{r}^{(n)}(u)}=-\xi_{r}(u)\left(1+f_{n+1}(u)-f_{n}(u)+O_{n}\left(\frac{1}{u^{2}}\right)\right) .
$$

La formule (30) permet alors de montrer le point (i). De même on a

$$
\begin{aligned}
\frac{d}{d u} \frac{\sigma_{r}^{(n+1)}(u)}{\sigma_{r}^{(n)}(u)} & =\frac{\sigma_{r}^{(n+2)}(u) \sigma_{r}^{(n)}(u)-\left(\sigma_{r}^{(n+1)}(u)\right)^{2}}{\left(\sigma_{r}^{(n)}(u)\right)^{2}} \\
& =\left(\xi_{r}(u)\right)^{2}\left(f_{n}(u)-2 f_{n+1}(u)+f_{n+2}(u)+O_{n}\left(\frac{1}{u^{2}}\right)\right) .
\end{aligned}
$$

La formule (30) permet alors d'estimer le facteur de $\left(\xi_{r}(u)\right)^{2}$ dans la dernière expression, et partant de démontrer le point (ii).

7. Démonstration du Théorème 2 et de son Corollaire. Le principe directeur de la démonstration étant partiellement caché par des problèmes techniques, nous pensons utile de le donner ici.

Schéma de la démonstration du Théorème 2. Nous spécifions $n=0$, le cas général se traitant de manière analogue. D'après la 
nouvelle définition de $\sigma$ (cf. (4)), on a

$$
\begin{aligned}
\sigma_{r}(u) & =r \varrho^{\prime}(u)+\int_{0}^{\infty} \varrho(u-r t) d \omega(t) \\
& =r \varrho^{\prime}(u)+\varrho(u) \int_{0}^{\infty} \exp \left\{\int_{u-r t}^{u}-\frac{\varrho^{\prime}(x)}{\varrho(x)} d x\right\} d \omega(t) .
\end{aligned}
$$

En première approximation, on a donc

$$
\begin{aligned}
\sigma_{r}(u) & \simeq r \varrho^{\prime}(u)+\varrho(u) \int_{0}^{\infty} e^{-r t \varrho^{\prime}(u) / \varrho(u)} d \omega(t) \\
& =\varrho(u) \exp \left\{-\gamma-I_{0}\left(-r \varrho^{\prime}(u) / \varrho(u)\right)\right\}
\end{aligned}
$$

d'après le Lemme 1(ii). Ce schéma de démonstration ne fonctionne efficacement que pour $r \log u \leq 1$. Dans le cas contraire, notre méthode se ramène pour l'essentiel à comparer les deux estimations du Théorème 1 correspondant à $\sigma_{r}(u)$ et à $\sigma_{0}(u)=e^{-\gamma} \varrho(u)$.

Démonstration du Théorème 2. Notre premier objectif est de montrer que

$$
\begin{aligned}
\varrho^{(n)}(u-t)=\varrho^{(n)}(u) e^{t l_{n}(u)}\left(1-\frac{t^{2}}{2} l_{n}^{\prime}(u)+\right. & \left.O_{n}\left(V_{n}(u, t)\right)\right) \\
& (n \geq 0, u>n+t>n)
\end{aligned}
$$

avec

et

$$
l_{n}(u)=l(u)=-\frac{\varrho^{(n+1)}(u)}{\varrho^{(n)}(u)}
$$

$$
V_{n}(u, t)=V= \begin{cases}\left(t^{3}+t^{4}\right) / u^{2} & (u \geq t+n+2), \\ t^{2} / u & (t+n+1 \leq u<t+n+2), \\ t & (t+n<u \leq t+n+1) .\end{cases}
$$

Supposons dans un premier temps que $t \geq \sqrt{u}$. Si $u \ll_{n} 1$, (35) est alors trivial. On peut donc se restreindre au cas où $u>c_{17}(n)$. On a alors en appliquant le Lemme 8(i) avec $r=0$

$$
e^{t l(u)} \geq \exp \left\{\int_{u-t}^{u} l(x) d x\right\} \asymp_{n} \frac{\varrho^{(n)}(u-t)}{\varrho^{(n)}(u)},
$$

ce qui suffit ici.

Supposons maintenant que $t<\sqrt{u}$. Il suffit alors d'analyser convenablement l'identité

$$
\varrho^{(n)}(u-t)=\varrho^{(n)}(u) \exp \left\{\int_{u-t}^{u} l(x) d x\right\} .
$$


On rappelle que $\varrho^{(n)}(u)$ est continue pour $u>n$ et dérivable pour $u>$ $n+1$. On exprime $l(x)$ par l'intermédiaire de la formule de Taylor en $u$. L'obtention de (35) résulte alors d'un banal calcul de développements limités.

Passons maintenant à la démonstration du Théorème 2 proprement dite. Pour cela on pose

$$
R=R(u, r)=\frac{\min ^{2}(1, r \log u)}{(\log u)(u \log u)^{1-2 r}}
$$

et on fixe un entier $n \geq 0$. Conformément aux hypothèses du théorème on suppose que

$$
u \geq n-r \log r .
$$

On distingue deux cas. Dans un premier temps, on suppose que

$$
r \xi_{0}(u) \leq \log \log 3 u .
$$

On a (cf. [15], III, 6, Corollaire 6.1)

$$
\omega^{\prime}(u) \ll \varrho^{\prime}(u) \ll u^{-u} \quad(u>1) .
$$

Cela permet de montrer, en dérivant l'équation (4), que

$$
\begin{aligned}
\sigma_{r}^{(n)}(u)=r \varrho^{(n+1)}(u)+ & \int_{0}^{(u-n) / r} \varrho^{(n)}(u-r t) d \omega(t) \\
& +O_{n}\left(\exp \left\{-\frac{u-n}{r} \log \left(\frac{u-n}{r}\right)\right\}\right) .
\end{aligned}
$$

Or d'après le cas $r=0$ du Théorème 1 ,

$$
\varrho^{(n)}(u)=\exp \left\{-u \log u-u \log \log 2 u+O_{n}(u)\right\} \quad(u>1),
$$

et d'après (38) et le Lemme 8(i)

$$
1 \ll_{n} \exp \left\{I_{0}(r l(u))\right\} \ll_{n} \exp \left\{c_{18} \log u / \log \log u\right\} .
$$

En utilisant de plus l'inégalité (37), le terme reste de (39) est donc

$$
\ll_{n} \varrho^{(n)}(u) \exp \left\{I_{0}(r l(u))\right\} R,
$$

ce qui est convenable.

Quant au terme principal dans (39), il est d'après (35)

$$
\begin{aligned}
=\varrho^{(n)}(u)\left[-r l(u)+\int_{0}^{(u-n) / r} e^{r t l(u)}(1\right. & -\frac{(r t)^{2} l^{\prime}(u)}{2} \\
& \left.\left.+O_{n}\left(V_{n}(u, r t)\right)\right) d \omega(t)\right] .
\end{aligned}
$$


D'après le Lemme 1(ii), on a

$$
-s+\int_{0}^{\infty} e^{s t} d \omega(t)=e^{-\gamma-I_{0}(s)}
$$

et en dérivant deux fois

$$
\int_{0}^{\infty} e^{s t} t^{2} d \omega(t)=e^{-\gamma-I_{0}(s)}\left(I_{0}^{\prime 2}(s)-I_{0}^{\prime \prime}(s)\right) .
$$

Le terme principal de (41) est donc

$$
\begin{aligned}
& =\varrho^{(n)}(u)\left[e^{-\gamma-I_{0}(r l(u))}\left(1-\frac{r^{2} l^{\prime}(u)}{2}\left(I_{0}^{\prime}(r l(u))-I_{0}^{\prime \prime 2}(r l(u))\right)\right)\right. \\
& \left.\quad+O_{n}\left(\int_{(u-n) / r}^{\infty} e^{r t l(u)} t^{2}\left|\omega^{\prime}(t)\right| d t\right)\right] .
\end{aligned}
$$

D'après (37), (38) et le Lemme 8(i), le terme reste de la dernière expression est

$$
\ll_{n} \varrho^{(n)}(u) \exp \left\{-\frac{u-n}{2 r} \log \frac{u-n}{r}\right\} \ll \varrho^{(n)}(u) R \exp \left\{-I_{0}(r l(u))\right\},
$$

ce qui est convenable.

Quant au terme principal, il est

$$
\begin{aligned}
& =\varrho^{(n)}(u) e^{-\gamma-I_{0}(r l(u))}\left(1+O_{n}\left(\frac{r^{2}}{u}\left(\frac{e^{r l(u)}-1}{r l(u)}\right)^{2}\right)\right) \\
& =\varrho^{(n)}(u) e^{-\gamma-I_{0}(r l(u))}\left(1+O_{n}(R)\right) .
\end{aligned}
$$

Pour achever la démonstration du Théorème 2 dans le cas (38), il suffit donc de majorer convenablement le terme reste issu de (41), soit, en omettant le facteur $\varrho^{(n)}(u)$,

$$
\int_{0}^{(u-n) / r} e^{r t l(u)} V_{n}(u, r t)|d \omega(t)| .
$$

On découpe l'intervalle d'intégration en trois sous-intervalles d'extrémités

$$
0, \quad \max \left(\frac{u-n-2}{r}, 0\right), \quad \max \left(\frac{u-n-1}{r}, 0\right), \quad \frac{u-n}{r} .
$$

On note $J_{1}, J_{2}$ et $J_{3}$ les intégrales correspondantes. On a

$$
J_{1} \ll \frac{r^{3}}{u^{2}} \int_{0}^{\infty} e^{r t l(u)}\left(1+t^{4}\right)|d \omega(t)| \ll \frac{r^{3}}{u^{2}}(1+\widehat{\varrho}(-r l(u))) \ll R e^{-I_{0}(r l(u))}
$$

d'après (40). 
Par (37), (40) et le Lemme 8(i), on a

$$
\begin{aligned}
J_{2} & \ll r^{2} \int_{\max ((u-n-2) / r, 0)}^{\max ((u-n-1) / r, 0)} e^{r t l(u)} t^{2}|d \omega(t)| \\
& \ll r^{2} e^{u l(u)} \exp \left\{-\max \left(\frac{u-n-2}{r}, 0\right) \log \left(\max \left(\frac{u-n-2}{r}, 0\right)\right)\right\} \\
& \ll R e^{-I_{0}(r l(u))}
\end{aligned}
$$

et

$$
\begin{aligned}
J_{3} & \ll r \int_{\max ((u-n-1) / r, 0)}^{(u-n) / r} e^{r t l(u)} t|d \omega(t)| \\
& \ll r e^{u l(u)} \exp \left\{-\max \left(\frac{u-n-1}{r}, 0\right) \log \left(\max \left(\frac{u-n-1}{r}, 0\right)\right)\right\} \\
& \ll \begin{cases}\operatorname{Re}^{-I_{0}(r l(u))} & (u \geq n+1-r \log r), \\
r & (n-r \log r \leq u<n+1-r \log r),\end{cases}
\end{aligned}
$$

ce qui permet de conclure le cas (38).

On suppose donc maintenant

$$
r \xi_{0}(u)>\log \log 3 u, \quad 0 \leq r \leq 1 / 2 .
$$

En appliquant le Théorème 1 pour $n=0$ et $n=1$ et en tenant compte des estimations

$$
\xi_{r}^{(k)}(u-h)=\xi_{r}^{(k)}(u)\left(1+O_{k}\left(\frac{1}{u}\right)\right) \quad(1+h \leq u, k \leq 1)
$$

qui découlent du Lemme 4(vii), on obtient pour $0 \leq t \leq 1$

$$
\sigma_{r}^{\prime}(u-t r)=-\left(1+O\left(\frac{1}{u}\right)\right) \xi_{r}(u) e^{t r \xi_{r}(u)} \sigma_{r}(u) .
$$

Or on a (cf. [8], Theorem 3)

$$
\frac{\partial}{\partial v}(v \sigma(u, v))=\sigma(u(1-1 / v), v-1)
$$

soit encore

$$
\frac{\partial}{\partial r} \sigma_{r}(u)=\frac{\sigma_{r}(u)-\sigma_{r}(u-r)}{r}=\int_{0}^{1} \sigma_{r}^{\prime}(u-t r) d t .
$$

En intégrant (43) on a donc

$$
-\frac{1}{\sigma_{r}(u)} \frac{\partial}{\partial r} \sigma_{r}(u)=\left(1+O\left(\frac{1}{u}\right)\right) \frac{e^{r \xi_{r}(u)}-1}{r} .
$$


Or d'après le Lemme 4(v),

$$
\begin{aligned}
\int_{0}^{r} \frac{e^{t \xi_{t}(u)}-1}{t} d t & =I_{0}\left(r \xi_{0}(u)\right)+\int_{0}^{r} \frac{e^{t \xi_{t}(u)}-e^{t \xi_{0}(u)}}{t} d t \\
& =I_{0}\left(r \xi_{0}(u)\right)+O(R),
\end{aligned}
$$

et $1 / u \ll R$ d'après (42). Donc en intégrant (44), on obtient

$$
\sigma_{r}(u)=\sigma_{0}(u) e^{-I_{0}\left(r \xi_{0}(u)\right)}(1+O(R)) .
$$

En procédant par récurrence et en utilisant le Lemme 8(i) et le Lemme 4(v), on en déduit que pour tout $n \geq 0$

$$
\sigma_{r}^{(n)}(u)=\sigma_{0}^{(n)}(u) e^{-I_{0}\left(r \xi_{0}(u)\right)}\left(1+O_{n}(R)\right) .
$$

Cela permet de conclure la démonstration du Théorème 2 puisque d'après le Lemme 8(i) et (42)

$$
I_{0}\left(r \xi_{0}(u)\right)-I_{0}(r l(u)) \ll_{n} \frac{r}{u} I_{0}^{\prime}\left(r \xi_{0}(u)\right) \asymp \frac{1}{(u \log u)^{1-r}} \ll R .
$$

Le Corollaire est une conséquence quasiment immédiate du Théorème 2. On suppose dans un premier temps que $0 \leq r \leq 1 / 2$. Quand $v \rightarrow+\infty$, on a $R \rightarrow 0$. Donc d'après le Théorème 2 , on a

$$
\sigma_{r}(u) \sim e^{-\gamma} \varrho(u) \Leftrightarrow I_{0}(r l(u)) \rightarrow 0 \Leftrightarrow r l(u) \rightarrow 0 \Leftrightarrow r \log u \rightarrow 0 .
$$

Comme (cf. [8], Theorem 1)

$$
\frac{u \partial \sigma}{\partial u}(u, v)+\sigma(u-1, v-v / u)=0
$$

et $\sigma \geq 0, \sigma$ est une fonction décroissante de $u$, à $v$ fixé.

Donc si $v \rightarrow+\infty$ et $r>1 / 2$, on a

$$
\sigma(u, v) \leq \sigma(u / 2, v)=o(\varrho(u))
$$

d'après le Théorème 2 appliqué au nouveau couple $(u / 2, v)$. Cela permet de conclure la démonstration du Corollaire.

8. Démonstration du Théorème 3. La démonstration est calquée sur celle des points (i) et (ii) du Lemme 4 de [12]. Le rôle de $\varrho$ est joué ici par la fonction $\sigma_{r}$. Comme pour $\Lambda(x, y)$ relativement à $\varrho$, le comportement de $M(x, y, z)$ pour $u$ petit dépend essentiellement de la proximité de $u$ avec les points de discontinuité des dérivées de $\sigma_{r}$.

Lemme 9. Soit $\varepsilon$ un réel $>0$ et $n$ un entier $\geq 0$. On a

$$
\int_{0}^{u} \sigma_{r}^{(n)}(t) y^{t} d t \ll_{n} x \frac{\sigma_{r}(u)(\log 2 u)^{n}}{\log y} \quad\left(\left(\mathrm{~L}_{\varepsilon}\right),\left(\mathrm{G}_{n, \varepsilon}\right)\right) .
$$


Démonstration. Ce résultat est le pendant de la formule (21) de [12]. On a

$$
\int_{0}^{\varepsilon u} \sigma_{r}^{(n)}(t) y^{t} d t \ll_{n} \frac{x^{\varepsilon}}{\log y} \ll_{n} x \frac{\sigma_{r}(u)(\log 2 u)^{n}}{\log y} \quad\left(\left(\mathrm{~L}_{\varepsilon}\right),\left(\mathrm{G}_{n, \varepsilon}\right)\right)
$$

d'après le Théorème 1. Pour majorer $\int_{\varepsilon u}^{u} \sigma_{r}^{(n)}(t) y^{t} d t$, on procède comme dans [12]. On compare $\sigma_{r}^{(n)}(t)$ à $\sigma_{r}^{(n)}(u)$ par l'intermédiaire de la formule

$$
\sigma_{r}^{(n)}(t)=\sigma_{r}^{(n)}(u) \exp \left\{-\int_{t}^{u}\left(\sigma_{r}^{(n+1)}(w) / \sigma_{r}^{(n)}(w)\right) d w\right\}
$$

en estimant l'intégrande à l'aide du Théorème 1. Nous omettons les détails.

La démonstration du Théorème 3 repose sur la formule suivante. On a d'après la formule de Mertens

$$
M(x, y, z)=(1+O(\exp \{-\sqrt{\log y}\})) e^{\gamma} \prod_{p \leq z}\left(1-\frac{1}{p}\right) \log y \int_{0}^{u} \sigma_{r}(t) y^{t} d t
$$

avec

$$
\begin{aligned}
(\log y) & \int_{0}^{u} \sigma_{r}(t) y^{t} d t=x \sum_{k=0}^{n} \frac{(-1)^{k} \sigma_{r}^{(k)}(u-0)}{(\log y)^{k}} \\
+ & \sum_{k=0}^{n} \frac{(-1)^{k+1}}{(\log y)^{k}} \sum_{\substack{i+j \leq k+1 \\
i+j r<u}}\left[\sigma_{r}^{(k)}(i+j r+0)-\sigma_{r}^{(k)}(i+j r-0)\right] y^{i} z^{j} \\
& +\frac{(-1)^{n+1}}{(\log y)^{n}} \int_{0}^{u} \sigma_{r}^{(n+1)}(t) y^{t} d t .
\end{aligned}
$$

Cette dernière formule se démontre aisément à l'aide d'intégrations par parties successives. En appliquant le Lemme 9 pour $n=0$, on voit que le terme d'erreur engendré par le terme $O(\exp \{-\sqrt{\log y}\})$ est convenable tant pour (i) que pour (ii).

On a $\sigma_{r}(r+0)-\sigma_{r}(r-0)=1$ et $\sigma_{r}(1+0)-\sigma_{r}(1-0)=-r$. Cela permet de démontrer le point (i) en appliquant la formule (47) pour $n=0$, conjointement avec le Lemme 9.

Montrons (ii). D'après le Lemme 9, l'intégrale du membre de droite de (47) est d'un ordre de grandeur convenable. Par ailleurs, d'après le Lemme 2, la somme double de $(47)$ est $\ll_{n} e^{d_{n}(x, y, z)}$. En discutant suivant le signe de $n+2-u$, on voit que cette dernière quantité est aussi d'un ordre de grandeur convenable. Cela montre (ii).

Pour démontrer le point (iii), il faut être plus précis. Sous les conditions $\left(\mathrm{L}_{\varepsilon}\right),\left(\mathrm{G}_{\varepsilon}\right)$ et $x \geq x_{0}(\varepsilon)$, on a d'après le Théorème $1,1+\sigma_{r}^{\prime}(u) /\left(\sigma_{r}(u) \log y\right)$ 
$\ll_{\varepsilon} 1$. Sous la condition supplémentaire $x \geq y^{2+\varepsilon}$, il découle du point (ii) que

$$
M(x, y, z)=e^{\gamma} \prod_{p \leq z}\left(1-\frac{1}{p}\right) x\left[\sigma_{r}(u)-\frac{\sigma_{r}^{\prime}(u)}{\log y}+O_{\varepsilon}\left(\sigma_{r}(u)\left(\frac{\log 2 u}{\log y}\right)^{2}\right)\right] .
$$

Cela suffit si $u \ll_{\varepsilon} 1$. On peut donc supposer dorénavant $u \geq 2 \varepsilon^{-1}$. Par (45) et (46) appliqués avec $n=0$, on est ramené à étudier

$$
J=\int_{0}^{(1-\varepsilon) u} \exp \left\{-\int_{u-t}^{u} \frac{\sigma_{r}^{\prime}(w)}{\sigma_{r}(w)} d w\right\} y^{-t} d t .
$$

D'après le Lemme 8(ii), on a pour $u \geq w \geq 2$

Comme $u \geq 2 \varepsilon^{-1}$, on a en substituant dans $J$,

$$
\frac{\sigma_{r}^{\prime}(w)}{\sigma_{r}(w)}=\frac{\sigma_{r}^{\prime}(u)}{\sigma_{r}(u)}+O\left(\frac{w-u}{u}\right) .
$$

$$
\begin{aligned}
J & =\int_{0}^{(1-\varepsilon) u} e^{-t \sigma_{r}^{\prime}(u) / \sigma_{r}(u)}\left(1+O\left(\frac{t^{2}}{u}\right)\right) y^{-t} d t \\
& =\frac{1+O(1 /(\log x \log y))}{\log y+\sigma_{r}^{\prime}(u) / \sigma_{r}(u)} .
\end{aligned}
$$

Cela permet d'achever le démonstration du point (iii) et partant, celle du Théorème 3 tout entier.

\section{Bibliographie}

[1] K. Alladi, The Turán-Kubilius inequality for integers without large factors, J. Reine Angew. Math. 335 (1982), 180-196.

[2] J. D. Bovey, On the size of prime factors of integers, Acta Arith. 33 (1977), 65-80.

[3] N. G. de Bruijn, On the number of uncancelled elements in the sieve of Eratosthenes, Nederl. Akad. Wetensch. Proc. 53 (1950), 803-812.

[4] - , The asymptotic behaviour of a function occurring in the theory of primes, J. Indian Math. Soc. (N.S.) 15 (1951), 25-32.

[5] A. A. Buchstab, An asymptotic estimate of a general number-theoretic function, Mat. Sb. 2 (44) (1937), 1239-1246 (in Russian).

[6] K. Dickman, On the frequency of numbers containing prime factors of a certain relative magnitude, Ark. Mat. Astr. Fys. 22 (1930), 1-14.

[7] E. Fouvry et G. Tenenbaum, Entiers sans grand facteur premier en progressions arithmétiques, Proc. London Math. Soc. 63 (1991), 449-494.

[8] J. B. Friedlander, Integers free from large and small primes, ibid. 33 (1976), 565-576.

[9] A. Hildebrand, Integers free of large prime factors and the Riemann hypothesis, Mathematika 31 (1984), 258-271. 
[10] A. Hildebrand, The asymptotic behaviour of the solutions of a class of differentialdifference equations, J. London Math. Soc. (2) 42 (1990), 11-31.

[11] A. Hildebrand and G. Tenenbaum, On integers free of large prime factors, Trans. Amer. Math. Soc. 296 (1986), 265-290.

[12] E. Saias, Sur le nombre des entiers sans grand facteur premier, J. Number Theory 32 (1989), 78-99.

[13] - Entiers sans grand ni petit facteur premier II, Acta Arith., à paraître.

[14] - Entiers sans grand ni petit facteur premier III, en préparation.

[15] G. Tenenbaum, Introduction à la théorie analytique et probabiliste des nombres, Institut Elie Cartan 13, Université de Nancy I, 1990.

[16] E. T. Whittaker and G. Watson, A Course of Modern Analysis, 4th ed., Cambridge University Press, 1927.

LABORATOIRE DE PROBABILITÉS

UNIVERSITÉ PARIS VI

4, PLACE JUSSIEU

75252 PARIS CEDEX 05, FRANCE 